\title{
新型含取代噻二唑环结构的吡唑肜类化合物的合成及生物活性研究
}

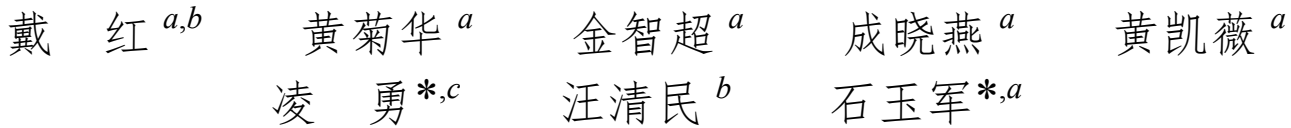 \\ ( ${ }^{a}$ 南通大学化学化工学院 南通 226019) \\ ( ${ }^{b}$ 南开大学元素有机化学国家重点实验室 天津 300071) \\ ( ${ }^{c}$ 南通大学药学院 南通 226001)
}

\begin{abstract}
摘要 为了从吡唑肜类化合物中寻找新的活性化合物, 通过活性亚结构拼接方法, 将取代噻二唑骨架引入到吡唑肜分 子中, 制备了一系列未见文献报道的新型含取代噻二唑环结构的吡唑肜类化合物. 采用核磁共振氢谱、碳谱和元素分 析确认了目标化合物的结构. 初步的生物活性测试结果表明, 在试验浓度下部分化合物对蚜虫、褐飞風表现出较好的 防治效果，部分化合物对粘虫显示出一定的杀虫活性.
\end{abstract}

关键词＼cjkstart取代噻二唑; 吡唑肜; 合成; 生物活性

\section{Synthesis and Bioactivities of Novel Pyrazole Oximes Containing Substituted Thiadiazole Moiety}

\author{
Dai, Hong ${ }^{a, b}$ \\ Huang, Juhua ${ }^{a}$ \\ Jin, Zhichao $^{a}$ \\ Cheng, Xiaoyan ${ }^{a}$ \\ Huang, Kaiwei ${ }^{a}$ \\ Ling, Yong ${ }^{*, c}$ \\ Wang, Qingmin ${ }^{b}$ \\ Shi, Yujun*,a \\ $\left({ }^{a}\right.$ College of Chemistry and Chemical Engineering, Nantong University, Nantong 226019) \\ $\left({ }^{b}\right.$ State Key Laboratory of Elemento-organic Chemistry, Nankai University, Tianjin 300071) \\ ( ${ }^{c}$ School of Pharmacy, Nantong University, Nantong 226001)
}

\begin{abstract}
In order to find novel potent pesticide from pyrazole oxime derivatives, a series of new pyrazole oximes carrying substituted thiadiazole moiety were prepared by the introduction of the key thiadiazole backbone into the molecules of pyrazole oximes. The structures of the title compounds were characterized by ${ }^{1} \mathrm{H}$ NMR, ${ }^{13} \mathrm{C}$ NMR and elemental analysis. Preliminary bioassay data displayed that some of the title compounds showed good insecticidal activities against Aphis craccivora and Nilaparvata lugens. In addition, some target compounds also exhibited certain larvicidal activity against Oriental armyworm. Keywords substituted thiadiazole; pyrazole oxime; synthesis; biological activity
\end{abstract}

目前，含吡唑肜结构的化合物因其广谱的杀虫、杀 螨、杀菌、抗病毒及抗癌等生物活性而成为新药创制研 究的热点课题之一 ${ }^{[1 \sim 5]}$. 近年来商品化的代表性化合物 有日本 Nihon Nohyaku 公司研发的杀螨剂唑螨酯, 该化 合物具有击倒快、持效长、受季节影响小等特点 ${ }^{[6]}$, 在 农作物保护方面扮演着十分重要的角色. 许多药物化学 工作者纷纷以唑螨酯为先导化合物，对其结构进行修饰
与改造, 合成出了一些具有良好生物活性的吡唑肜类衍 生物 ${ }^{[7 \sim 10]}$. 最近，戴红等 ${ }^{[11 \sim 14]}$ 设计并制备了含吡啶、噻 唑等杂环结构的吡唑肜类化合物，其中部分化合物表现 出较好的杀虫、杀螨、植物生长调节或杀菌活性. 噻二 唑类衍生物亦为含氮杂环体系中的重要一员，在农药和 医药领域有着广泛的应用, 不少噻二唑类化合物具有优 良的杀菌 ${ }^{[15]} 、$ 杀虫 ${ }^{[16]} 、$ 除草 ${ }^{[17,18]} 、$ 抗病毒 ${ }^{[19]}$ 、植物生长

\footnotetext{
*E-mail: zhanghj2801@163.com, yjshi2015@163.com

Received August 18, 2015; revised September 11, 2015; published online September 17, 2015.

Project supported by the National Natural Science Foundation of China (Nos. 21202089, 21372135), the Open Project Fund of State Key Laboratory of Elemento-organic Chemistry (No. 201412), the Science and Technology Innovation Foundation for the College Students of Jiangsu Province and the Science and Technology Project Fund of Nantong City (Nos. AS2013004, AS2014011).

国家自然科学基金(Nos. 21202089, 21372135)、元素有机化学国家重点实验室开放课题(No. 201412)、江苏省大学生创新训练计划和南通市科技计划 (Nos. AS2013004, AS2014011)资助项目.
} 


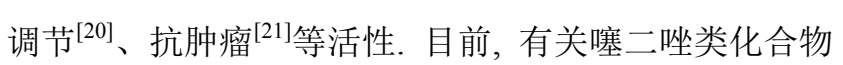
分子的合理设计、合成与生物活性研究仍为药物化学研 究的重要领域之一 ${ }^{[22 ~ 24]}$. 此外, 近年来亚甲氧基苯甲基 结构单元也常作为良好药效团引入到母体化合物分子 结构中, 以改善其生物活性. 2010 年 Wang 等 ${ }^{[25]}$ 报道了 一系列含噻唑连亚甲氧基苯甲基结构的氰基丙烯酸酯类 化合物, 其中部分化合物表现出较好的除草活性. 2015 年吴珊珊等 ${ }^{[26]}$ 合成了一系列含吡啶连亚甲氧基苯甲基单 元结构的氰基丙烯酸酯类衍生物, 某些化合物显示出优 良的除草活性. 为了寻找新的含吡唑肜杂环结构的先导 化合物, 我们采用活性亚结构拼接的方法, 将噻二唑环 连亚甲氧基苯甲基活性单元引入到吡唑肜结构中, 设计 合成了一系列未见文献报道的新型含取代噻二唑环结构 的吡唑肜类化合物. 利用 ${ }^{1} \mathrm{H} N \mathrm{NM},{ }^{13} \mathrm{C} \mathrm{NMR}$ 及元素分析 对目标化合物的结构进行了表征. 初步生物活性测试结 果显示, 部分化合物在试验浓度下对蚜虫和褐飞虫具有 良好的杀灭效果, 部分化合物还对粘虫表现出一定的杀 虫活性. 目标化合物的合成路线如 Scheme 1 所示.

\section{1 结果与讨论}

\section{1 化合物的合成}

在目标化合物的合成中, 我们以化合物 $7 \mathbf{d}$ 为研究 对象，尝试了不同的反应条件对其反应收率的影响. 最初选用无水碳酸钠作缚酸剂, 以丙酮作溶剂, 尝试了 加热回流 $10 \mathrm{~h}$ 的方法, TLC 跟踪有新点生成, 大部分原 料仍未发生反应，化合物 7d 的收率较低，仅为 $20 \%$; 当
换用无水碳酸钾作缚酸剂，以丙酮作溶剂时，探讨了加 热回流 $10 \mathrm{~h}$ 的方法, TLC 跟踪有新点产生, 化合物 7d 的 收率为 $29 \%$; 后来换用沸点较高的乙腈或 DMF 作溶剂, 加热回流 $10 \mathrm{~h}, \mathrm{TLC}$ 跟踪发现均有新点产生, 化合物 7d 的收率分别为 $45 \%$ 和 $41 \%$; 最后选用无水碳酸钾作缚酸 剂, 加入催化量的碳酸铯, 以乙腈作溶剂, 加热回流 10 $\mathrm{h}$, 化合物 7d 的收率达到 $56 \%$, 较前面几种方法有了明 显的提高. 碳酸铯在反应过程中起到了催化作用, 其原 因可能是由于铯是碱金属中原子半径最大的元素，其盐 碳酸铯在乙腈溶剂中具有较好的溶解性, 进而使反应更 易进行. 最终采用该合成方法顺利地合成了 16 个含取 代噻二唑环结构的新型吡唑肜类化合物.

\section{2 化合物的图谱分析}

我们以目标物 7e 的核磁氢谱与碳谱数据为例进行 解析. $\delta 7.79$ 处的单峰对应于 $\mathrm{CH}=\mathrm{N}$ 氢的吸收峰; $\delta 7.43$ 处的双重峰对应于 4-溴取代基苯环上两个氢的吸收峰; $\delta 7.22$ 处的双重峰对应于与噻二唑甲氧基相连苯环上两 个氢的吸收峰; $\delta 6.95$ 处的双重峰对应于与噻二唑甲氧 基相连苯环上另外两个氢的吸收峰; $\delta 6.79$ 处的双重峰 对应于 4-溴取代基苯环上另外两个氢的吸收峰; $\delta 5.31$ 处的单峰为与噻二唑环相连的亚甲基两个氢的吸收峰; $\delta 4.93$ 处的单峰为与苯环相连的亚甲基两个氢的吸收 峰; $\delta 4.61$ 处的四重峰为与乙氧基上亚甲基两个氢的吸 收峰; $\delta 3.63$ 处的单峰对应于吡唑环 1-位 N-甲基三个氢 的吸收峰; $\delta 2.37$ 处的单峰对应于吡唑环 3-位甲基三个 氢的吸收峰; $\delta 1.49$ 处的三重峰为乙氧基上甲基三个氢

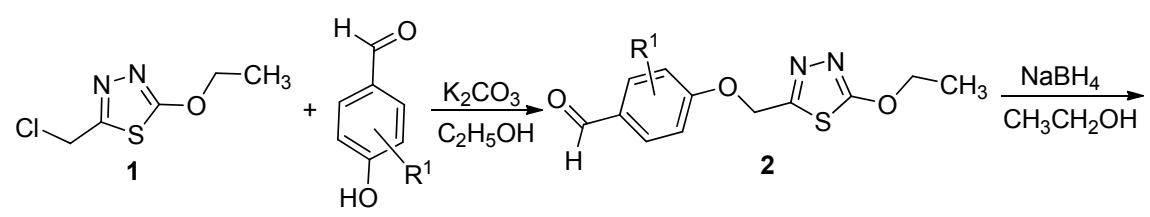<smiles>[R1]c1cc(OCc2nnc(OCC)s2)ccc1OCc1nnc(OCC)s1</smiles>

3<smiles>[R]c1ccc(Oc2c(C=O)c(C)nn2C)cc1</smiles><smiles>[R]c1cccc(Oc2c(/C=N/O)c(C)nn2C)c1</smiles>

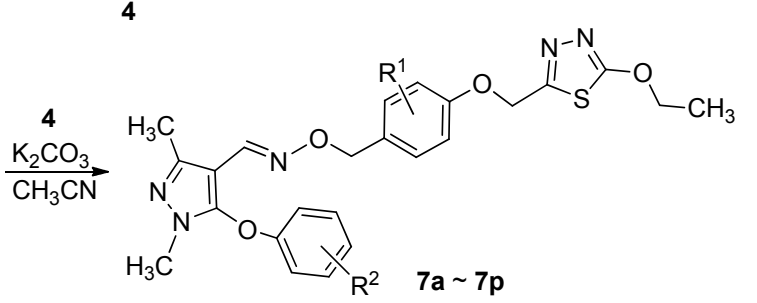

7a: $R^{1}=H, R^{2}=2-F ; 7 b: R^{1}=H, R^{2}=3-F ; 7 c: R^{1}=H, R^{2}=3-C l ; 7 d: R^{1}=H, R^{2}=4-C l ; 7 e: R^{1}=H, R^{2}=4-B r ;$ 7f: $R^{1}=H, R^{2}=4-l ; 7 g: R^{1}=H, R^{2}=3-C F_{3} ; 7 h: R^{1}=H, R^{2}=3-C_{3} ; 7 i: R^{1}=3-O_{3} H_{3}, R^{2}=4-F ; 7 j: R^{1}=3-O_{3}$, $\mathrm{R}^{2}=4-\mathrm{Cl} ; \mathbf{7 k}: \mathrm{R}^{1}=3-\mathrm{OCH}_{3}, \mathrm{R}^{2}=2,4-\mathrm{Cl}_{2} ; 7 \mathrm{II}: \mathrm{R}^{1}=3-\mathrm{OCH}_{3}, \mathrm{R}^{2}=4-\mathrm{CH}_{3} ; 7 \mathrm{~m}: \mathrm{R}^{1}=3-\mathrm{OCH}_{3}, \mathrm{R}^{2}=4-\mathrm{OCF}_{3}$; $7 n: R^{1}=3-C l, R^{2}=4-F ; 70: R^{1}=3-C l, R^{2}=4-C_{3} ; 7 p: R^{1}=3-C l, R^{2}=4-O_{3}$

图式 1 含取代噻二唑环结构的吡唑肜类化合物的合成

Scheme 1 Synthesis of pyrazole oximes containing substituted thiadiazole moiety 
的吸收峰; $\delta 154.7$ 处的峰为 $\mathrm{CH}=\mathrm{N}$ 碳原子的信号峰; $\delta$ $74.6,68.4,64.3$ 处的峰分别对应于三个亚甲基碳原子的 信号峰; $\delta 33.2$ 处的峰为吡唑环 1-位 $\mathrm{N}$-甲基碳原子的信 号峰; $\delta 13.4$ 处的峰为吡唑环 3-位甲基碳原子的信号峰; $\delta 13.2$ 处的峰为乙氧基上甲基碳原子的信号峰.

\section{3 化合物的生物活性分析}

目标化合物 7a $\sim 7 \mathbf{p}$ 对蚜虫(Aphis craccivora)、朱砂 叶螨(Tetranychus cinnabarinus)、褐飞䖝(Nilaparvata lugens)和粘虫(Oriental armyworm)的生物活性测试结果 见表 1. 初步的生测结果显示, 部分目标化合物表现出 较好的杀蚜虫活性. 其中化合物 7b $\sim 7 \mathbf{j}, 7 \mathbf{0}$ 和 7p 在测试 浓度为 $500 \mu \mathrm{g} / \mathrm{mL}$ 时对蚜虫的杀死率分别为 $100 \%, 80 \%$, $100 \%, 100 \%, 100 \%, 100 \%, 90 \%$ 和 $100 \%$, 当测试剂量降 至 $100 \mu \mathrm{g} / \mathrm{mL}$ 时, 7b, 7e, 7f 和 7p 对蚜虫仍具有良好的防 治效果，其抑制率分别为 $90 \%, 90 \%, 100 \%$ 和 100\%. 部 分目标物对褐飞虫表现出一定的杀灭效果. 其中化合物 $7 \mathbf{b}, 7 \mathbf{d}, 7 \mathbf{e}, 7 \mathbf{g}$ 和 7p 在测试浓度为 $500 \mu \mathrm{g} / \mathrm{mL}$ 时对褐飞 禹的杀死率分别为 $100 \%, 100 \%, 100 \%, 100 \%$ 和 $80 \%$. 部 分目标物对粘虫还显示出一定的杀虫活性. 其中化合物 $7 \mathbf{d}, 7 \mathrm{e}$ 和 7j 在测试浓度为 $500 \mu \mathrm{g} / \mathrm{mL}$ 时对褐飞虫的杀死 率分别为 $80 \%, 85 \%$ 和 $80 \%$. 此外, 所有目标物在测试浓 度为 $500 \mu \mathrm{g} / \mathrm{mL}$ 时对朱砂叶螨均未表现出杀螨虫活性. 从以上实验数据可看出, 将取代噻二唑结构单元引入到 吡唑肜类化合物分子中, 所得的某些目标物对蚜虫、褐 飞虫具有较好的防治效果, 某些化合物对粘虫还表现出
一定的杀虫活性. 该结构类型化合物可作进一步的修饰 与优化，以期今后能够发现生物活性更好的化合物.

\section{2 结论}

设计合成了 16 个新型含取代噻二唑杂环结构的吡 唑肜类衍生物. 通过核磁共振氢谱、碳谱及元素分析等 手段表征了其目标化合物的结构. 初步的生物活性测试 结果显示, 某些目标物对蚛虫、褐飞虫具有较好的防治 效果. 另外, 部分化合物对粘虫还表现出一定的杀虫活 性. 这些实验结果为今后继续从事吡唑肜类化合物的研 究提供了重要数据.

\section{3 实验部分}

\section{1 仪器与试剂}

X-4 型数字显示熔点测定仪(北京泰克仪器有限公 司), 温度计未经校正; Yanaco-CHN CORDER MT-3 自 动元素分析仪; Bruker AM-400 型核磁共振仪, 以 $\mathrm{CDCl}_{3}$ 为溶剂, $\mathrm{TMS}$ 为内标; 柱层析硅胶为 $\mathrm{H}$ 型 (青岛海洋化工 厂，200 300 目). 所用试剂均为分析纯. 中间体 2-氯甲 基-5-乙氧基-1,3,4-噻二唑(1)参照文献[27]方法合成. 中 间体 4-[(5-乙氧基-1,3,4-噻二唑-2-基)甲氧基]芳甲醛(2) 参照文献[25]方法合成. 中间体 1-甲基-3-甲基-5-芳氧基 吡唑-4-甲醛(5)和 1-甲基-3-甲基-5-芳氧基吡唑-4-甲醛肜 (6)参照文献[28]方法合成.

表 1 目标化合物 $7 \mathbf{a} \sim 7 \mathbf{p}$ 的杀虫活性(死亡率/\%) $)^{a}$

Table 1 Insecticidal activities (mortality/\%) of target compounds $\mathbf{7 a} \sim \mathbf{7 p}$

\begin{tabular}{|c|c|c|c|c|c|c|c|}
\hline \multirow{2}{*}{ Compd. } & \multirow{2}{*}{$\mathrm{R}^{1}$} & \multirow{2}{*}{$\mathrm{R}^{2}$} & \multicolumn{2}{|c|}{ Aphis Craccivora } & \multicolumn{2}{|c|}{$\begin{array}{c}\text { Nilaparvata lugens } \\
\end{array}$} & \multirow{2}{*}{$\begin{array}{c}\text { Oriental Armyworm } \\
500 \mu \mathrm{g} / \mathrm{mL}\end{array}$} \\
\hline & & & $500 \mu \mathrm{g} / \mathrm{mL}$ & $100 \mu \mathrm{g} / \mathrm{mL}$ & $500 \mu \mathrm{g} / \mathrm{mL}$ & $100 \mu \mathrm{g} / \mathrm{mL}$ & \\
\hline $7 \mathbf{a}$ & $\mathrm{H}$ & $2-\mathrm{F}$ & 0 & - & 0 & - & 0 \\
\hline $7 b$ & $\mathrm{H}$ & $3-\mathrm{F}$ & 100 & 90 & 100 & 0 & 0 \\
\hline $7 \mathrm{c}$ & $\mathrm{H}$ & $3-\mathrm{Cl}$ & 80 & 0 & 50 & - & 0 \\
\hline $7 d$ & $\mathrm{H}$ & $4-\mathrm{Cl}$ & 100 & 0 & 100 & 0 & 80 \\
\hline $7 e$ & $\mathrm{H}$ & $4-\mathrm{Br}$ & 100 & 90 & 100 & 0 & 85 \\
\hline $7 f$ & $\mathrm{H}$ & $4-\mathrm{I}$ & 100 & 100 & 50 & - & 0 \\
\hline $7 \mathrm{~g}$ & $\mathrm{H}$ & $3-\mathrm{CF}_{3}$ & 0 & - & 100 & 0 & 0 \\
\hline $7 \mathrm{~h}$ & $\mathrm{H}$ & $3-\mathrm{CH}_{3}$ & 0 & - & 0 & - & 0 \\
\hline $7 \mathbf{i}$ & $3-\mathrm{OCH}_{3}$ & $4-\mathrm{F}$ & 0 & - & 0 & - & 0 \\
\hline $7 \mathbf{j}$ & $3-\mathrm{OCH}_{3}$ & $4-\mathrm{Cl}$ & 100 & 0 & 0 & - & 80 \\
\hline $7 \mathbf{k}$ & $3-\mathrm{OCH}_{3}$ & $2,4-\mathrm{Cl}_{2}$ & 0 & - & 0 & - & 0 \\
\hline 71 & $3-\mathrm{OCH}_{3}$ & $4-\mathrm{CH}_{3}$ & 0 & - & 0 & - & 0 \\
\hline $7 m$ & $3-\mathrm{OCH}_{3}$ & $4-\mathrm{OCF}_{3}$ & 0 & - & 0 & - & 0 \\
\hline $7 n$ & $3-\mathrm{Cl}$ & $4-\mathrm{F}$ & 0 & - & 0 & - & 0 \\
\hline 70 & $3-\mathrm{Cl}$ & $4-\mathrm{CH}_{3}$ & 90 & 50 & 50 & - & 0 \\
\hline $7 p$ & $3-\mathrm{Cl}$ & $4-\mathrm{OCH}_{3}$ & 100 & 100 & 80 & 0 & 0 \\
\hline Imidacloprid & & & 100 & 100 & 100 & 100 & - \\
\hline Pyridalyl & & & - & - & - & - & 100 \\
\hline
\end{tabular}




\subsection{4 -[(5-乙氧基-1,3,4-噻二唑-2-基)甲氧基]芳甲醇(3) 的合成}

在 $100 \mathrm{~mL}$ 圆底烧瓶中, 加入 $3 \mathrm{mmol} 4$-[(5-乙氧基1,3,4-噻二唑-2-基)甲氧基]芳甲醛(2)及 $30 \mathrm{~mL}$ 无水乙醇, 冰浴条件下向其中分批加入 $6 \mathrm{mmol}$ 嗍氢化钠固体, 加 毕, 向反应液中加入催化量的碘, 室温搅拌 $2 \sim 3 \mathrm{~h}$, 停 止反应. 向反应液中加入 $20 \mathrm{~mL}$ 水, 用稀盐酸调节 $\mathrm{pH}$ $=4 \sim 5$, 静置, 析出大量固体, 经抽滤后得到中间体 $\mathbf{3}$, 产品可直接用于后面的反应. 为了确证中间体 $\mathbf{3}$ 的结构, 选取其中取代基为 $\mathrm{H}$ 的化合物为代表, 经过柱层析 $[V$ (石油醚) $: V$ (乙酸乙酯 $)=8: 1]$ 分离纯化, 得黄色固 体 3, 产率 65\%. m.p. $76 \sim 78{ }^{\circ} \mathrm{C} ;{ }^{1} \mathrm{H}$ NMR $(400 \mathrm{MHz}$, $\left.\mathrm{CDCl}_{3}\right) \delta: 1.47\left(\mathrm{t}, J=7.2 \mathrm{~Hz}, 3 \mathrm{H}, \mathrm{CH}_{3}\right), 4.59$ (q, $J=7.2$ $\left.\mathrm{Hz}, 2 \mathrm{H}, \mathrm{CH}_{2}\right), 4.63$ (s, 2H, $\mathrm{CH}_{2}$ ), 5.27 (s, $\left.2 \mathrm{H}, \mathrm{CH}_{2}\right), 6,97$ (d, $J=8.4 \mathrm{~Hz}, 2 \mathrm{H}, \mathrm{ArH}), 7.31$ (d, $J=8.4 \mathrm{~Hz}, 2 \mathrm{H}, \mathrm{ArH})$; ${ }^{13} \mathrm{C}$ NMR (100 MHz, $\left.\mathrm{CDCl}_{3}\right) \delta: 14.3,64.7,65.3,69.5$, $114.9,128.7,134.7,157.0,160.8,176.5$. Anal. calcd for $\mathrm{C}_{12} \mathrm{H}_{14} \mathrm{~N}_{2} \mathrm{O}_{3} \mathrm{~S}$ : C 54.12, H 5.30, N 10.52; found C 54.26, H 5.18, N 10.33,

\subsection{4-[(5-乙氧基-1,3,4-噻二唑-2-基)甲氧基]芳甲基氯 (4)的合成}

在 $100 \mathrm{~mL}$ 圆底烧瓶中, 加入 $10 \mathrm{mmol}$ 醇中间体 $\mathbf{3}$ 及 $25 \mathrm{~mL}$ 二氯甲烷, 室温搅拌下向其中加入 $10 \mathrm{mmol}$ 二 氯亚砜, 加毕, 将反应液摚拌 $8 \sim 15 \mathrm{~min}$, 将反应液倒入 盛有适量水的烧杯中, 静置, 分离出有机相, 无水硫酸 钠干燥, 脱溶得到相应的关键中间体 4 , 不经提纯可直 接用于下一步反应, 为了验证中间体 $\mathbf{3}$ 的结构, 我们选 取取代基为 $\mathrm{H}$ 的化合物为代表, 经过柱层析 $[V($ 石油 醚) $: V$ (乙酸乙酯 $)=10: 1$ ]分离纯化, 得黄色固体 4 , 产 率 58\%. m.p. 90 92 ${ }^{\circ} \mathrm{C} ;{ }^{1} \mathrm{H}$ NMR $\left(400 \mathrm{MHz}, \mathrm{CDCl}_{3}\right) \delta$ : $1.47\left(\mathrm{t}, J=7.2 \mathrm{~Hz}, 3 \mathrm{H}, \mathrm{CH}_{3}\right), 4.56 \sim 4.61(\mathrm{~m}, 4 \mathrm{H}, 2 \times$ $\left.\mathrm{CH}_{2}\right), 5.30\left(\mathrm{~s}, 2 \mathrm{H}, \mathrm{CH}_{2}\right), 6.97(\mathrm{~d}, \quad J=8.0 \mathrm{~Hz}, 2 \mathrm{H}, \mathrm{ArH})$, $7.32(\mathrm{~d}, J=8.4 \mathrm{~Hz}, 2 \mathrm{H}, \operatorname{ArH}) ;{ }^{13} \mathrm{C}$ NMR $(100 \mathrm{MHz}$, $\left.\mathrm{CDCl}_{3}\right) \delta: 14.4,45.9,65.4,69.5,115.1,130.2,131.1$, 157.5, 160.4, 176.5. Anal. calcd for $\mathrm{C}_{12} \mathrm{H}_{13} \mathrm{ClN}_{2} \mathrm{O}_{2} \mathrm{~S}$ : C 50.61, H 4,.60, N 9.84; found C 50.43, H 4.76, N 9.71,

\section{4 目标化合物 7 的合成}

在 $100 \mathrm{~mL}$ 圆底烧瓶中, 加入 $2 \mathrm{mmol}$ 1-甲基-3-甲 基-5-取代苯氧基吡唑-4-甲醛肜(6)、3 mmol 中间体 4、4 $\mathrm{mmol}$ 无水碳酸钾及 $30 \mathrm{~mL}$ 乙腈, 室温搅拌下, 向其中 加入催化量的碳酸铯. 加毕, 将反应液加热回流 8 17 h. 停止加热, 抽滤, 脱溶, 所得残余物以 $V$ (石油醚) $: V($ 乙 酸乙酯) $=20: 1$ 为洗脱剂进行柱层析分离, 得到目标化 合物 $\mathbf{7 a} \sim \mathbf{7 p}$.
1-甲基-3-甲基-5-(2-氟苯氧基)吡唑-4-甲醛- $O$ - $\{[4$ (5-乙氧基-1,3,4-噻二唑-2-基)甲氧基]苯甲基 $\}$ 肜(7a): 淡 黄色固体, 产率 $46 \%$. m.p. 83 $85{ }^{\circ} \mathrm{C} ;{ }^{1} \mathrm{H}$ NMR (400 $\left.\mathrm{MHz}, \mathrm{CDCl}_{3}\right) \delta: 1.48\left(\mathrm{t}, J=7.2 \mathrm{~Hz}, 3 \mathrm{H}, \mathrm{CH}_{3}\right), 2.36(\mathrm{~s}, 3 \mathrm{H}$, $\mathrm{CH}_{3}$ ), 3.67 (s, 3H, N-CH$)_{3}$ ), 4.61 (q, J=7.2 Hz, $2 \mathrm{H}, \mathrm{CH}_{2}$ ), $4.92\left(\mathrm{~s}, 2 \mathrm{H}, \mathrm{CH}_{2}\right), 5.30\left(\mathrm{~s}, 2 \mathrm{H}, \mathrm{CH}_{2}\right), 6.75 \sim 6.79(\mathrm{~m}, 1 \mathrm{H}$, ArH), $6.93 \sim 7.10(\mathrm{~m}, 4 \mathrm{H}, \mathrm{ArH}), 7.17 \sim 7.22(\mathrm{~m}, 1 \mathrm{H}, \mathrm{ArH})$, $7.24(\mathrm{~d}, J=8.8 \mathrm{~Hz}, 2 \mathrm{H}, \mathrm{ArH}), 7.79(\mathrm{~s}, 1 \mathrm{H}, \mathrm{CH}=\mathrm{N}) ;{ }^{13} \mathrm{C}$ NMR $\left(100 \mathrm{MHz}, \mathrm{CDCl}_{3}\right) \delta: 13.4,13.5,33.2,64.4,68.4$, 74.6, 99.0, 113.7, 115.7, 116.0, 116.2, 123.4, 123.5, 123.6, $129.3,130.1,139.2,145.9,149.7,152.2,156.2,159.7$, 175.5. Anal. calcd for $\mathrm{C}_{24} \mathrm{H}_{24} \mathrm{FN}_{5} \mathrm{O}_{4} \mathrm{~S}$ : C 57.94, H 4.86, N 14.08; found C 57.83, H 4.98, N 14.25.

1-甲基-3-甲基-5-(3-氟苯氧基)吡唑-4-甲醛- $O$ - $\{[4-$ (5-乙氧基-1,3,4-噻二唑-2-基)甲氧基]苯甲基 $\}$ 肜 (7b) : 淡 黄色固体, 产率 $48 \%$. m.p. $69 \sim 71{ }^{\circ} \mathrm{C} ;{ }^{1} \mathrm{H}$ NMR (400 $\left.\mathrm{MHz}, \mathrm{CDCl}_{3}\right) \delta: 1.48\left(\mathrm{t}, J=7.2 \mathrm{~Hz}, 3 \mathrm{H}, \mathrm{CH}_{3}\right), 2.38(\mathrm{~s}, 3 \mathrm{H}$, $\left.\mathrm{CH}_{3}\right), 3.63$ (s, $\left.3 \mathrm{H}, \mathrm{N}-\mathrm{CH}_{3}\right), 4.61$ (q, $J=7.2 \mathrm{~Hz}, 2 \mathrm{H}, \mathrm{CH}_{2}$ ), $4.94\left(\mathrm{~s}, 2 \mathrm{H}, \mathrm{CH}_{2}\right), 5.30\left(\mathrm{~s}, 2 \mathrm{H}, \mathrm{CH}_{2}\right), 6.62 \sim 6.70(\mathrm{~m}, 2 \mathrm{H}$, $\operatorname{ArH}), 6.80 \sim 6.86(\mathrm{~m}, 1 \mathrm{H}, \operatorname{ArH}), 6.94(\mathrm{~d}, J=8.4 \mathrm{~Hz}, 2 \mathrm{H}$, $\mathrm{ArH}), 7.23 \sim 7.27(\mathrm{~m}, 3 \mathrm{H}, \mathrm{ArH}), 7.81(\mathrm{~s}, 1 \mathrm{H}, \mathrm{CH}=\mathrm{N}) ;{ }^{13} \mathrm{C}$ NMR $\left(100 \mathrm{MHz}, \mathrm{CDCl}_{3}\right) \delta: 14.4,14.5,34.2,65.4,69.4$, $75.6,100.5,103.4,103.7,110.6,110.8,110.9,114.7$, $130.3,130.8,130.9,131.1,140.2,147.0,157.3,157.7$, 160.8, 176.5. Anal. calcd for $\mathrm{C}_{24} \mathrm{H}_{24} \mathrm{FN}_{5} \mathrm{O}_{4} \mathrm{~S}$ : C 57.94, $\mathrm{H}$ 4.86, N 14.08; found C 58.05, H 4.69, N 13.92 .

1-甲基-3-甲基-5-(3-氯苯氧基)吡唑-4-甲醛- $O$ - $\{[4-$ (5-乙氧基-1,3,4-噻二唑-2-基)甲氧基]苯甲基 $\}$ 肜 (7c) : 淡 黄色固体, 产率 $45 \%$. m.p. $53 \sim 55{ }^{\circ} \mathrm{C} ;{ }^{1} \mathrm{H}$ NMR (400 $\left.\mathrm{MHz}, \mathrm{CDCl}_{3}\right) \delta: 1.44$ (t, $\left.J=7.2 \mathrm{~Hz}, 3 \mathrm{H}, \mathrm{CH}_{3}\right), 2.33(\mathrm{~s}, 3 \mathrm{H}$, $\left.\mathrm{CH}_{3}\right), 3.58\left(\mathrm{~s}, 3 \mathrm{H}, \mathrm{NCH}_{3}\right), 4.56\left(\mathrm{q}, J=7.2 \mathrm{~Hz}, 2 \mathrm{H}, \mathrm{CH}_{2}\right)$, 4.89 (s, $\left.2 \mathrm{H}, \mathrm{CH}_{2}\right), 5.26\left(\mathrm{~s}, 2 \mathrm{H}, \mathrm{CH}_{2}\right), 6.73 \sim 6.91(\mathrm{~m}, 4 \mathrm{H}$, $\mathrm{ArH}), 7.06 \sim 7.20(\mathrm{~m}, 4 \mathrm{H}, \mathrm{ArH}), 7.76(\mathrm{~s}, 1 \mathrm{H}, \mathrm{CH}=\mathrm{N}) ;{ }^{13} \mathrm{C}$ NMR $\left(100 \mathrm{MHz}, \mathrm{CDCl}_{3}\right) \delta: 14.4,14.5,34.2,65.4,69.4$, $75.6,100.5,113.6,114.7,116.0,124.0,130.3,130.7$, $131.1,135.5,140.2,146.8,147.0,157.2,157.3,160.8$, 176.5. Anal. calcd for $\mathrm{C}_{24} \mathrm{H}_{24} \mathrm{ClN}_{5} \mathrm{O}_{4} \mathrm{~S}$ : C 56.08, $\mathrm{H} 4.71, \mathrm{~N}$ 13.63; found C 56.23, H 4.58, N 13.51 .

1-甲基-3-甲基-5-(4-氯苯氧基)吡唑-4-甲醛- $O$ - $\{[4-$ (5-乙氧基-1,3,4-噻二唑-2-基)甲氧基]苯甲基 $\}$ 肜(7d)：淡 黄色固体, 产率 56\%. m.p. 105 107 ${ }^{\circ} \mathrm{C} ;{ }^{1} \mathrm{H}$ NMR $(400$ $\left.\mathrm{MHz}, \mathrm{CDCl}_{3}\right) \delta: 1.49\left(\mathrm{t}, J=7.2 \mathrm{~Hz}, 3 \mathrm{H}, \mathrm{CH}_{3}\right), 2.37(\mathrm{~s}, 3 \mathrm{H}$, $\left.\mathrm{CH}_{3}\right), 3.63$ (s, $3 \mathrm{H}, \mathrm{N}-\mathrm{CH}_{3}$ ), 4.61 (q, $J=7.2 \mathrm{~Hz}, 2 \mathrm{H}, \mathrm{CH}_{2}$ ), $4.93\left(\mathrm{~s}, 2 \mathrm{H}, \mathrm{CH}_{2}\right), 5.31\left(\mathrm{~s}, 2 \mathrm{H}, \mathrm{CH}_{2}\right), 6.84(\mathrm{~d}, J=8.8 \mathrm{~Hz}$, $2 \mathrm{H}, \operatorname{ArH}), 6.94$ (d, $J=8.4 \mathrm{~Hz}, 2 \mathrm{H}, \operatorname{ArH}), 7.21 \sim 7.29$ (m, 
4H, $\operatorname{ArH}), 7.79(\mathrm{~s}, 1 \mathrm{H}, \mathrm{CH}=\mathrm{N}) ;{ }^{13} \mathrm{C}$ NMR $(100 \mathrm{MHz}$, $\left.\mathrm{CDCl}_{3}\right) \delta: 14.4,14.5,34.2,65.4,69.4,75.6,100.3,114.7$, $116.6,128.7,129.9,130.3,131.1,140.3,147.0,147.1$, 155.2, 157.3, 160.7, 176.5. Anal. calcd for $\mathrm{C}_{24} \mathrm{H}_{24} \mathrm{ClN}_{5} \mathrm{O}_{4} \mathrm{~S}$ : C 56.08, H 4.71, N 13.63; found C 56.19, H 4.60, N 13.46.

1-甲基-3-甲基-5-(4-溴苯氧基)吡唑-4-甲醛- $O$ - $\{[4-$ (5-乙氧基-1,3,4-噻二唑-2-基)甲氧基]苯甲基 $\}$ 肜 (7e): 淡 黄色固体, 产率 53\%. m.p. 84 86 ${ }^{\circ} \mathrm{C}$; ${ }^{1} \mathrm{H}$ NMR (400 $\left.\mathrm{MHz}, \mathrm{CDCl}_{3}\right) \delta: 1.49\left(\mathrm{t}, J=7.2 \mathrm{~Hz}, 3 \mathrm{H}, \mathrm{CH}_{3}\right), 2.37(\mathrm{~s}, 3 \mathrm{H}$, $\mathrm{CH}_{3}$ ), 3.63 (s, $3 \mathrm{H}, \mathrm{N}-\mathrm{CH}_{3}$ ), 4.61 (q, J=7.2 Hz, $2 \mathrm{H}, \mathrm{CH}_{2}$ ), $4.93\left(\mathrm{~s}, 2 \mathrm{H}, \mathrm{CH}_{2}\right), 5.31\left(\mathrm{~s}, 2 \mathrm{H}, \mathrm{CH}_{2}\right), 6.79(\mathrm{~d}, J=9.2 \mathrm{~Hz}$, 2H, ArH), 6.95 (d, $J=8.8 \mathrm{~Hz}, 2 \mathrm{H}, \operatorname{ArH}), 7.22$ (d, $J=8.8$ $\mathrm{Hz}, 2 \mathrm{H}, \mathrm{ArH}), 7.43$ (d, $J=8.8 \mathrm{~Hz}, 2 \mathrm{H}, \mathrm{ArH}), 7.79$ (s, $1 \mathrm{H}$, $\mathrm{CH}=\mathrm{N}) ;{ }^{13} \mathrm{C}$ NMR $\left(100 \mathrm{MHz}, \mathrm{CDCl}_{3}\right) \delta: 13.2,13.4,33.2$, 64.3, 68.4, 74.6, 99.3, 113.7, 115.1, 116.0, 129.3, 130.1, $131.8,139.2,145.9,146.0,154.7,156.2,159.7,175.5$. Anal. calcd for $\mathrm{C}_{24} \mathrm{H}_{24} \mathrm{BrN}_{5} \mathrm{O}_{4} \mathrm{~S}$ : C 51.62, H 4.33, N 12.54; found $\mathrm{C} 51.43, \mathrm{H} 4.51, \mathrm{~N} 12.70$.

1-甲基-3-甲基-5-(4-碘苯氧基)吡唑-4-甲醛- $O$ - $\{[4$ (5-乙氧基-1,3,4-噻二唑-2-基)甲氧基]苯甲基 $\}$ 肜 (7f) : 淡 黄色固体, 产率 50\%. m.p. $65 \sim 67{ }^{\circ} \mathrm{C} ;{ }^{1} \mathrm{H}$ NMR (400 $\left.\mathrm{MHz}, \mathrm{CDCl}_{3}\right) \delta: 1.46$ (t, $\left.J=7.2 \mathrm{~Hz}, 3 \mathrm{H}, \mathrm{CH}_{3}\right), 2.34$ (s, $3 \mathrm{H}$, $\left.\mathrm{CH}_{3}\right), 3.59\left(\mathrm{~s}, 3 \mathrm{H}, \mathrm{N}-\mathrm{CH}_{3}\right), 4.59$ (q, $J=7.2 \mathrm{~Hz}, 2 \mathrm{H}, \mathrm{CH}_{2}$ ), $4.90\left(\mathrm{~s}, 2 \mathrm{H}, \mathrm{CH}_{2}\right), 5.30$ (s, $\left.2 \mathrm{H}, \mathrm{CH}_{2}\right), 6.65$ (d, $J=8.8 \mathrm{~Hz}$, $2 \mathrm{H}, \operatorname{ArH}), 6.93$ (d, $J=8.8 \mathrm{~Hz}, 2 \mathrm{H}, \mathrm{ArH}), 7.20$ (d, $J=8.8$ $\mathrm{Hz}, 2 \mathrm{H}, \mathrm{ArH}), 7.59$ (d, J=8.4 Hz, 2H, ArH), 7.77 (s, $1 \mathrm{H}$, $\mathrm{CH}=\mathrm{N}) ;{ }^{13} \mathrm{C}$ NMR $\left(100 \mathrm{MHz}, \mathrm{CDCl}_{3}\right) \delta: 14.4,14.5,34.2$, 65.4, 69.4, 75.6, 86.4, 100.3, 114.8, 117.5, 128.8, 130.4, $131.1,138.8,140.3,146.9,147.1,156.6,157.3,160.8$, 176.5. Anal. calcd for $\mathrm{C}_{24} \mathrm{H}_{24} \mathrm{IN}_{5} \mathrm{O}_{4} \mathrm{~S}: \mathrm{C} 47.61, \mathrm{H} 4.00, \mathrm{~N}$ 11.57; found C 47.42, H 4.19, N 11.73.

1-甲基-3-甲基-5-(3-三氟甲基苯氧基)吡唑-4-甲醛$O$ - $\{[4-$-(5-乙氧基-1,3,4-噻二唑-2-基)甲氧基]苯甲基 $\}$ 肜 (7g): 淡黄色固体, 产率 47\%. m.p. 50 52 ${ }^{\circ} \mathrm{C} ;{ }^{1} \mathrm{H}$ NMR $\left(400 \mathrm{MHz}, \mathrm{CDCl}_{3}\right) \delta: 1.46\left(\mathrm{t}, J=7.2 \mathrm{~Hz}, 3 \mathrm{H}, \mathrm{CH}_{3}\right), 2.36$ (s, $\left.3 \mathrm{H}, \mathrm{CH}_{3}\right), 3.62\left(\mathrm{~s}, 3 \mathrm{H}, \mathrm{N}-\mathrm{CH}_{3}\right), 4.59(\mathrm{q}, J=7.2 \mathrm{~Hz}, 2 \mathrm{H}$, $\left.\mathrm{CH}_{2}\right), 4.86\left(\mathrm{~s}, 2 \mathrm{H}, \mathrm{CH}_{2}\right), 5.28$ (s, $\left.2 \mathrm{H}, \mathrm{CH}_{2}\right), 6.90$ (d, $J=8.4$ $\mathrm{Hz}, 2 \mathrm{H}, \mathrm{ArH}), 7.00 \sim 7.19(\mathrm{~m}, 4 \mathrm{H}, \mathrm{ArH}), 7.36 \sim 7.44(\mathrm{~m}$, $2 \mathrm{H}, \mathrm{ArH}), 7.78(\mathrm{~s}, 1 \mathrm{H}, \mathrm{CH}=\mathrm{N}) ;{ }^{13} \mathrm{C}$ NMR $(100 \mathrm{MHz}$, $\left.\mathrm{CDCl}_{3}\right) \delta: 14.3,14.4,34.3,65.3,69.4,75.6,100.4,112.7$, $114.7,115.0,118.3,120.4,128.7,130.2,130.6,131.0$, 140.1, 146.5, 147.2, 156.7, 157.2, 160.8, 176.5. Anal. calcd for $\mathrm{C}_{25} \mathrm{H}_{24} \mathrm{~F}_{3} \mathrm{~N}_{5} \mathrm{O}_{4} \mathrm{~S}$ : C 54.84, $\mathrm{H} 4.42, \mathrm{~N} 12.79$; found $\mathrm{C}$ $54.98, \mathrm{H} 4.31, \mathrm{~N} 12.66$.

1- 甲基-3-甲基-5-(3-甲基苯氧基)吡唑-4-甲醛- $O$ - \{[4-(5-乙氧基-1,3,4-噻二唑-2-基)甲氧基]苯甲基\}肜(7h): 淡黄色固体, 产率 $45 \%$. m.p. $62 \sim 64{ }^{\circ} \mathrm{C}$; $1 \mathrm{H}$ NMR (400 $\left.\mathrm{MHz}, \mathrm{CDCl}_{3}\right) \delta: 1.48\left(\mathrm{t}, J=7.2 \mathrm{~Hz}, 3 \mathrm{H}, \mathrm{CH}_{3}\right), 2.33(\mathrm{~s}, 3 \mathrm{H}$, Ar- $\left.\mathrm{CH}_{3}\right), 2.41$ (s, $\left.3 \mathrm{H}, \mathrm{CH}_{3}\right), 3.62\left(\mathrm{~s}, 3 \mathrm{H}, \mathrm{NCH}_{3}\right), 4.61$ (q, $\left.J=7.2 \mathrm{~Hz}, 2 \mathrm{H}, \mathrm{CH}_{2}\right), 4.96\left(\mathrm{~s}, 2 \mathrm{H}, \mathrm{CH}_{2}\right), 5.30\left(\mathrm{~s}, 2 \mathrm{H}, \mathrm{CH}_{2}\right)$, 6.69 (d, $J=7.2 \mathrm{~Hz}, 2 \mathrm{H}, \mathrm{ArH}), 6.92 \sim 6.95(\mathrm{~m}, 3 \mathrm{H}, \mathrm{ArH})$, $7.18 \sim 7.22(\mathrm{~m}, 1 \mathrm{H}, \mathrm{ArH}), 7.26$ (d, $J=8.8 \mathrm{~Hz}, 2 \mathrm{H}, \operatorname{ArH})$, $7.79(\mathrm{~s}, 1 \mathrm{H}, \mathrm{CH}=\mathrm{N}) ;{ }^{13} \mathrm{C}$ NMR $\left(100 \mathrm{MHz}, \mathrm{CDCl}_{3}\right) \delta$ : 14.1, 14.4, 21.4, 29.7, 65.4, 69.4, 75.6, 100.4, 112.3, 114.7, $115.9,124.6,129.7,130.4,131.1,140.4,140.6,146.8$, 148.0, 156.7, 157.3, 160.8, 176.5. Anal. calcd for $\mathrm{C}_{25} \mathrm{H}_{27}$ $\mathrm{N}_{5} \mathrm{O}_{4} \mathrm{~S}: \mathrm{C} 60.83, \mathrm{H} 5.51, \mathrm{~N} 14.19$; found C 60.70, H 5.65, $\mathrm{N} 14.36$.

1- 甲基-3-甲基-5-(4-氟苯氧基)吡唑-4-甲醛- $O$ \{[4-(5-乙氧基-1,3,4-噻二唑-2-基)甲氧基]-(3-甲氧基)苯 甲基\}肜(7i): 淡黄色固体，产率 51\%. m.p. $86 \sim 88{ }^{\circ} \mathrm{C}$; ${ }^{1} \mathrm{H}$ NMR $\left(400 \mathrm{MHz}, \mathrm{CDCl}_{3}\right) \delta: 1.47(\mathrm{t}, J=7.2 \mathrm{~Hz}, 3 \mathrm{H}$, $\mathrm{CH}_{3}$ ), 2.38 (s, 3H, $\mathrm{CH}_{3}$ ), 3.62 (s, 3H, N-CH $\mathrm{CH}_{3}, 3.89$ (s, $3 \mathrm{H}$, Ar- $\left.-\mathrm{OCH}_{3}\right), 4.59$ (q, $\left.J=7.2 \mathrm{~Hz}, 2 \mathrm{H}, \mathrm{CH}_{2}\right), 4.93(\mathrm{~s}, 2 \mathrm{H}$, $\left.\mathrm{CH}_{2}\right), 5.36\left(\mathrm{~s}, 2 \mathrm{H}, \mathrm{CH}_{2}\right), 6.81 \sim 6.91(\mathrm{~m}, 4 \mathrm{H}, \mathrm{ArH}), 6.96 \sim$ $7.03(\mathrm{~m}, 3 \mathrm{H}, \mathrm{ArH}), 7.81(\mathrm{~s}, 1 \mathrm{H}, \mathrm{CH}=\mathrm{N}) ;{ }^{13} \mathrm{C} \mathrm{NMR}(100$ $\left.\mathrm{MHz}, \mathrm{CDCl}_{3}\right) \delta: 14.4,14.7,34.2,55.9,66.8,69.4,75.9$, $100.6,112.6,115.0,116.4,116.5,116.6,121.2,132.2$, $140.6,146.7,147.0,147.7,149.7,152.7,157.5,159.9$, 161.1, 176.6. Anal. calcd for $\mathrm{C}_{25} \mathrm{H}_{26} \mathrm{FN}_{5} \mathrm{O}_{5} \mathrm{~S}: \mathrm{C} 56.92, \mathrm{H}$ 4.97, N 13.27; found C 56.98, H 4.79, N 13.17.

1-甲基-3-甲基-5-(4-氯苯氧基)吡唑-4-甲醛- $O$ \{[4-(5-乙氧基-1,3,4-噻二唑-2-基)甲氧基]-(3-甲氧基)苯 甲基\}肜 $(7 \mathbf{j})$ : 淡黄色固体，产率 59\%. m.p. $85 \sim 87{ }^{\circ} \mathrm{C}$; ${ }^{1} \mathrm{H}$ NMR $\left(400 \mathrm{MHz}, \mathrm{CDCl}_{3}\right) \delta: 1.48(\mathrm{t}, J=7.2 \mathrm{~Hz}, 3 \mathrm{H}$, $\left.\mathrm{CH}_{3}\right), 2.38\left(\mathrm{~s}, 3 \mathrm{H}, \mathrm{CH}_{3}\right), 3.62\left(\mathrm{~s}, 3 \mathrm{H}, \mathrm{N}-\mathrm{CH}_{3}\right), 3.88(\mathrm{~s}, 3 \mathrm{H}$, $\left.\mathrm{Ar}-\mathrm{OCH}_{3}\right), 4.60\left(\mathrm{q}, J=7.2 \mathrm{~Hz}, 2 \mathrm{H}, \mathrm{CH}_{2}\right), 4.93(\mathrm{~s}, 2 \mathrm{H}$, $\left.\mathrm{CH}_{2}\right), 5.37\left(\mathrm{~s}, 2 \mathrm{H}, \mathrm{CH}_{2}\right), 6.80 \sim 6.91(\mathrm{~m}, 4 \mathrm{H}, \mathrm{ArH}), 6.97(\mathrm{~d}$, $J=8.0 \mathrm{~Hz}, 1 \mathrm{H}, \mathrm{ArH}), 7.27 \sim 7.29(\mathrm{~m}, 2 \mathrm{H}, \mathrm{ArH}), 7.81(\mathrm{~s}$, $1 \mathrm{H}, \mathrm{CH}=\mathrm{N}) ;{ }^{13} \mathrm{C} \mathrm{NMR}\left(100 \mathrm{MHz}, \mathrm{CDCl}_{3}\right) \delta: 14.4,14.6$, 34.2, 55.9, 66.8, 69.4, 75.9, 100.2, 112.6, 115.0, 116.6, $121.2,128.7,129.9,132.2,140.4,146.7,147.0,147.2$, 149.7, 155.3, 161.1, 176.6. Anal. calcd for $\mathrm{C}_{25} \mathrm{H}_{26} \mathrm{ClN}_{5} \mathrm{O}_{5} \mathrm{~S}$ : C 55.19, H 4.82, N 12.87; found C 55.32, H 4.65, N 12.68.

1-甲基-3-甲基-5-(2,4-二氯苯氧基)吡唑-4-甲醛- $O$ \{[4-(5-乙氧基-1,3,4-噻二唑-2-基)甲氧基]-(3-甲氧基)苯 甲基\}肪(7k): 白色固体，产率 52\%. m.p. 104 106 ${ }^{\circ} \mathrm{C}$; ${ }^{1} \mathrm{H}$ NMR $\left(400 \mathrm{MHz}, \mathrm{CDCl}_{3}\right) \delta: 1.46(\mathrm{t}, J=7.2 \mathrm{~Hz}, 3 \mathrm{H}$, $\mathrm{CH}_{3}$ ), 2.34 (s, $3 \mathrm{H}, \mathrm{CH}_{3}$ ), 3.64 (s, $3 \mathrm{H}, \mathrm{NCH}_{3}$ ), 3.86 (s, $3 \mathrm{H}$, $\left.\mathrm{Ar}-\mathrm{OCH}_{3}\right), 4.58\left(\mathrm{q}, J=7.2 \mathrm{~Hz}, 2 \mathrm{H}, \mathrm{CH}_{2}\right), 4.88(\mathrm{~s}, 2 \mathrm{H}$, 
$\left.\mathrm{CH}_{2}\right), 5.34\left(\mathrm{~s}, 2 \mathrm{H}, \mathrm{CH}_{2}\right), 6.60(\mathrm{~d}, J=9.2 \mathrm{~Hz}, 1 \mathrm{H}, \mathrm{ArH})$, 6.76 7.12 (m, 4H, ArH), 7.44 (s, 1H, ArH), $7.78(\mathrm{~s}, 1 \mathrm{H}$, $\mathrm{CH}=\mathrm{N}) ;{ }^{13} \mathrm{C}$ NMR $\left(100 \mathrm{MHz}, \mathrm{CDCl}_{3}\right) \delta: 14.3,14.4,34.2$, 55.9, 66.8, 69.4, 75.9, 100.1, 112.5, 115.0, 116.3, 121.1, $123.6,127.9,129.1,130.5,132.2,140.1,146.6,146.7$, 147.1, 149.7, 150.9, 161.1, 176.6. Anal. calcd for $\mathrm{C}_{25} \mathrm{H}_{25^{-}}$ $\mathrm{Cl}_{2} \mathrm{~N}_{5} \mathrm{O}_{5} \mathrm{~S}: \mathrm{C} 51.91, \mathrm{H} 4.36, \mathrm{~N} 12.11$; found $\mathrm{C} 51.76, \mathrm{H}$ 4.52, N 12.30 .

1-甲基-3-甲基-5-(4-甲基苯氧基)吡唑-4-甲醛- $O$ \{[4-(5-乙氧基-1,3,4-噻二唑-2-基)甲氧基]-(3-甲氧基)苯 甲基\}肜(71): 淡黄色固体, 产率 $61 \%$. m.p. $77 \sim 79{ }^{\circ} \mathrm{C}$; ${ }^{1} \mathrm{H}$ NMR $\left(400 \mathrm{MHz}, \mathrm{CDCl}_{3}\right) \delta: 1.45(\mathrm{t}, J=7.2 \mathrm{~Hz}, 3 \mathrm{H}$, $\mathrm{CH}_{3}$ ), 2.30 (s, 3H, Ar- $\mathrm{CH}_{3}$ ), 2.37 (s, $\left.3 \mathrm{H}, \mathrm{CH}_{3}\right), 3.58$ (s, $3 \mathrm{H}$, $\left.\mathrm{N}-\mathrm{CH}_{3}\right), 3.86$ (s, 3H, Ar-OCH $\left.{ }_{3}\right), 4.57$ (q, J=7.2 Hz, 2H, $\left.\mathrm{CH}_{2}\right), 4.94\left(\mathrm{~s}, 2 \mathrm{H}, \mathrm{CH}_{2}\right), 5.34\left(\mathrm{~s}, 2 \mathrm{H}, \mathrm{CH}_{2}\right), 6.75 \sim 6.96(\mathrm{~m}$, $5 \mathrm{H}, \mathrm{ArH}), 7.08$ (d, $J=8.4 \mathrm{~Hz}, 2 \mathrm{H}, \mathrm{ArH}), 7.79$ (s, $1 \mathrm{H}$, $\mathrm{CH}=\mathrm{N}) ;{ }^{13} \mathrm{C}$ NMR $\left(100 \mathrm{MHz}, \mathrm{CDCl}_{3}\right) \delta: 14.4,15.0,20.6$, $34.2,55.9,66.8,69.4,75.9,100.1,112.6,115.1,121.3$, $130.4,132.3,133.2,141.0,146.6,146.8,148.2,149.7$, 154.8, 161.1, 176.6. Anal. calcd for $\mathrm{C}_{26} \mathrm{H}_{29} \mathrm{~N}_{5} \mathrm{O}_{5} \mathrm{~S}$ : C 59.64, H 5.58, N 13.38; found C 59.52, H 5.73, N 13.52.

1-甲基-3-甲基-5-(4-三氟甲氧基苯氧基)吡唑-4-甲 醛- $O$ - $\{[4$-(5-乙氧基-1,3,4-噻二唑-2-基)甲氧基]-(3-甲氧 基)苯甲基\}肟(7m): 淡黄色固体, 产率 54\%. m.p. 46 $48{ }^{\circ} \mathrm{C} ;{ }^{1} \mathrm{H}$ NMR $\left(400 \mathrm{MHz}, \mathrm{CDCl}_{3}\right) \delta: 1.46(\mathrm{t}, J=7.2 \mathrm{~Hz}$, $\left.3 \mathrm{H}, \mathrm{CH}_{3}\right), 2.37$ (s, 3H, $\mathrm{CH}_{3}$ ), 3.62 (s, $\left.3 \mathrm{H}, \mathrm{N}-\mathrm{CH}_{3}\right), 3.87$ (s, $\left.3 \mathrm{H}, \mathrm{Ar}-\mathrm{OCH}_{3}\right), 4.58$ (q, J=7.2 Hz, 2H, $\mathrm{CH}_{2}$ ), 4.90 (s, $2 \mathrm{H}$, $\left.\mathrm{CH}_{2}\right), 5.34\left(\mathrm{~s}, 2 \mathrm{H}, \mathrm{CH}_{2}\right), 6.80 \sim 6.92(\mathrm{~m}, 4 \mathrm{H}, \mathrm{ArH}), 6.96(\mathrm{~d}$, $J=8.0 \mathrm{~Hz}, 2 \mathrm{H}, \mathrm{ArH}), 7.18$ (d, $J=8.8 \mathrm{~Hz}, 2 \mathrm{H}, \mathrm{ArH}), 7.81$ $(\mathrm{s}, 1 \mathrm{H}, \mathrm{CH}=\mathrm{N}) ;{ }^{13} \mathrm{C}$ NMR $(100 \mathrm{MHz}, \mathrm{CDCl} 3): \delta 14.4$, 14.6, 34.2, 55.9, 66.8, 69.4, 75.9, 100.2, 112.6, 115.1, $116.4,119.1,121.2,121.7,122.8,124.3,132.2,140.4$, 144.8, 146.7, 147.2, 149.7, 155.0, 158.8, 169.8. Anal. calcd for $\mathrm{C}_{26} \mathrm{H}_{26} \mathrm{~F}_{3} \mathrm{~N}_{5} \mathrm{O}_{6} \mathrm{~S}$ : C 52.61, H 4.42, N 11.80; found $\mathrm{C}$ $52.80, \mathrm{H} 4.25, \mathrm{~N} 11.69$.

1-甲基-3-甲基-5-(4-氟苯氧基)吡唑-4-甲醛- $O$ \{[4-(5-乙氧基-1,3,4-噻二唑-2-基)甲氧基]-(3-氯)苯甲基 $\}$ 肜(7n): 淡黄色固体, 产率 $43 \%$. m.p. $103 \sim 105{ }^{\circ} \mathrm{C} ;{ }^{1} \mathrm{H}$ NMR (400 MHz, $\left.\mathrm{CDCl}_{3}\right) \delta: 1.47$ (t, $J=7.2 \mathrm{~Hz}, 3 \mathrm{H}, \mathrm{CH}_{3}$ ), $2.35\left(\mathrm{~s}, 3 \mathrm{H}, \mathrm{CH}_{3}\right), 3.61\left(\mathrm{~s}, 3 \mathrm{H}, \mathrm{N}-\mathrm{CH}_{3}\right), 4.59(\mathrm{q}, J=7.2 \mathrm{~Hz}$, $\left.2 \mathrm{H}, \mathrm{CH}_{2}\right), 4.88$ (s, $\left.2 \mathrm{H}, \mathrm{CH}_{2}\right), 5.35$ (s, $\left.2 \mathrm{H}, \mathrm{CH}_{2}\right), 6.82 \sim 7.13$ (m, 6H, ArH), $7.34(\mathrm{~s}, 1 \mathrm{H}, \mathrm{ArH}), 7.77(\mathrm{~s}, 1 \mathrm{H}, \mathrm{CH}=\mathrm{N}) ;{ }^{13} \mathrm{C}$ NMR $\left(100 \mathrm{MHz}, \mathrm{CDCl}_{3}\right) \delta: 14.4,14.6,34.2,66.4,69.5$, $74.8,100.0,114.0,116.4,116.5,116.6,123.1,128.1$, $130.3,130.8,132.6,140.8,147.0,147.7,152.6,152.7$,
157.5, 159.9, 176.7. Anal. calcd for $\mathrm{C}_{24} \mathrm{H}_{23} \mathrm{ClFN}_{5} \mathrm{O}_{4} \mathrm{~S}$ : C 54.18, H 4.36, N 13.16; found C 54.32, H 4.23, N 13.01.

1-甲基-3-甲基-5-(4-甲基苯氧基)吡唑-4-甲醛- $O$ \{[4-(5-乙氧基-1,3,4-噻二唑-2-基)甲氧基]-(3-氯)苯甲基 $\}$ 肜(7o): 淡黄色固体，产率 51\%. m.p. $88 \sim 90{ }^{\circ} \mathrm{C} ; 1 \mathrm{H}$ NMR (400 MHz, $\left.\mathrm{CDCl}_{3}\right) \delta: 1.49$ (t, $J=7.2 \mathrm{~Hz}, 3 \mathrm{H}, \mathrm{CH}_{3}$ ), $2.33\left(\mathrm{~s}, 3 \mathrm{H}, \mathrm{Ar}-\mathrm{CH}_{3}\right), 2.38\left(\mathrm{~s}, 3 \mathrm{H}, \mathrm{CH}_{3}\right), 3.62(\mathrm{~s}, 3 \mathrm{H}$, $\left.\mathrm{N}-\mathrm{CH}_{3}\right), 4.61$ (q, $\left.J=7.2 \mathrm{~Hz}, 2 \mathrm{H}, \mathrm{CH}_{2}\right), 4.92\left(\mathrm{~s}, 2 \mathrm{H}, \mathrm{CH}_{2}\right)$, $5.37\left(\mathrm{~s}, 2 \mathrm{H}, \mathrm{CH}_{2}\right), 6.79(\mathrm{~d}, J=8.8 \mathrm{~Hz}, 2 \mathrm{H}, \mathrm{ArH}), 7.01(\mathrm{~d}$, $J=8.4 \mathrm{~Hz}, 1 \mathrm{H}, \mathrm{ArH}), 7.12$ (d, $J=8.4 \mathrm{~Hz}, 2 \mathrm{H}, \mathrm{ArH}), 7.16$ (d, $J=8.4 \mathrm{~Hz}, 1 \mathrm{H}, \operatorname{ArH}), 7.38(\mathrm{~s}, 1 \mathrm{H}, \operatorname{ArH}), 7.78(\mathrm{~s}, 1 \mathrm{H}$, $\mathrm{CH}=\mathrm{N}) ;{ }^{13} \mathrm{C}$ NMR $\left(100 \mathrm{MHz}, \mathrm{CDCl}_{3}\right) \delta: 14.4,14.8,20.6$, $34.2,66.4,69.5,74.8,100.0,114.0,115.1,123.2,128.1$, $130.4,132.7,133.2,141.0,146.8,148.2,152.7,154.7$, 160.2, 176.7. Anal. calcd for $\mathrm{C}_{25} \mathrm{H}_{26} \mathrm{ClN}_{5} \mathrm{O}_{4} \mathrm{~S}: \mathrm{C} 56.87, \mathrm{H}$ 4.96, N 13.26; found C 56.72, H 5.08, N 13.43 .

1-甲基-3-甲基-5-(4-甲氧基苯氧基)吡唑-4-甲醛- $O$ \{[4-(5-乙氧基-1,3,4-噻二唑-2-基)甲氧基]-(3-氯)苯甲基\} 肜(7p): 白色固体, 产率 53\%. m.p. 67 69 ${ }^{\circ} \mathrm{C} ;{ }^{1} \mathrm{H}$ NMR $\left(400 \mathrm{MHz}, \mathrm{CDCl}_{3}\right) \delta: 1.47\left(\mathrm{t}, J=7.2 \mathrm{~Hz}, 3 \mathrm{H}, \mathrm{CH}_{3}\right), 2.35$ (s, $\left.3 \mathrm{H}, \mathrm{CH}_{3}\right), 3.60$ (s, $\left.3 \mathrm{H}, \mathrm{N}-\mathrm{CH}_{3}\right), 3.78$ (s, $\left.3 \mathrm{H}, \mathrm{Ar}-\mathrm{OCH}_{3}\right)$, 4.59 (q, $\left.J=6.8 \mathrm{~Hz}, 2 \mathrm{H}, \mathrm{CH}_{2}\right), 4.90\left(\mathrm{~s}, 2 \mathrm{H}, \mathrm{CH}_{2}\right), 5.35$ (s, $\left.2 \mathrm{H}, \mathrm{CH}_{2}\right), 6.82$ (s, 4H, ArH), 6.98 (d, J=8.4 Hz, 1H, ArH), 7.14 (d, J=8.4 Hz, 1H, ArH), 7.37 (s, 1H, ArH), 7.76 (s, $1 \mathrm{H}, \mathrm{CH}=\mathrm{N}) ;{ }^{13} \mathrm{C}$ NMR $\left(100 \mathrm{MHz}, \mathrm{CDCl}_{3}\right) \delta: 14.4,14.8$, $34.2,55.7,66.4,69.5,74.8,99.8,114.0,114.9,116.3$, $123.1,128.1,130.9,132.7,141.1,146.8,148.5,150.6$, 152.7, 155.8, 160.2, 176.7. Anal. calcd for $\mathrm{C}_{25} \mathrm{H}_{26} \mathrm{ClN}_{5} \mathrm{O}_{5} \mathrm{~S}$ : C 55.19, H 4.82, N 12.87; found C 55.03, H 4.96, N 13.02.

\section{5 目标化合物的生物活性测试}

杀虫活性测试所选昆虫分别为蚜虫(Aphis craccivora)、朱砂叶螨(Tetranychus cinnabarinus)、褐飞虫 (Nilaparvata lugens)和粘虫(Oriental armyworm). 对照药 剂分别为吡虫啉(Imidacloprid)、唑螨酯(Fenpyroximate) 和啶虫丙醚(Pyridalyl). 杀虫试验方法分别如下:

药剂配置: 对于化合物, 用分析天平称取一定质量 的原药, 用含吐温-80 乳化剂的 DMF 溶解配制成 $1 \%$ 母 液, 然后用蒸馏水稀释即得到 $500 \mu \mathrm{g} / \mathrm{mL}$ 的药液, 其它 浓度的药液可由此进行稀释. 每个处理 3 次重复, 设空白 对照.

蚜虫、螨虫和褐飞虫: 采用喷雾法. 首先, 分别将接 有蚜虫、朱砂叶螨的蚕豆叶片和接有褐飞虫的水稻苗于 Potter 喷雾塔下喷雾处理, 处理后朱砂叶螨和褐飞闽置 于 $24 \sim 27{ }^{\circ} \mathrm{C}$ 观察室内培养, 蚜虫置于 $20 \sim 22{ }^{\circ} \mathrm{C}$ 观察 室内培养, $2 \mathrm{~d}$ 后观察结果, 检查死活虫数, 并进行统计 
分析.

粘虫: 采用浸叶碟法. 首先, 将适量玉米叶在配好 的药液中充分浸润后自然阴干, 放入垫有滤纸的培养血 中, 接粘虫 3 龄中期幼虫 10 头/血, 置于 $24 \sim 27{ }^{\circ} \mathrm{C}$ 观察 室内培养, 调查药后 $2 \mathrm{~d}$ 的死活虫数, 并进行统计分析.

辅助材料(Supporting Information) 化合物 $7 \mathbf{a} \sim 7 \mathbf{p}$ 的 ${ }^{1} \mathrm{H}$ NMR 和 ${ }^{13} \mathrm{C}$ NMR 图谱. 这些材料可以免费从本刊网 站(http://sioc-journal,cn/)上下载.

\section{References}

[1] Motoba, K.; Nishizawa, H.; Suzuki, T.; Hamaguchi, H.; Uchida, M.; Funayama, S. Pestic. Biochem. Physiol. 2000, 67, 73.

[2] Park, H. J.; Lee, K.; Park, S. J.; Ahn, B.; Lee, J. C.; Cho, H. Y.; Lee, K. I. Bioorg. Med. Chem. Lett. 2005, 15, 3307.

[3] Li, Y.; Zhang, H. Q.; Liu, J.; Yang, X. P.; Liu, Z. J. J. Agric. Food Chem. 2006, 54, 3636.

[4] Chen, L.; Ou, X. M.; Mao, C. H.; Shang, J.; Huang, R. Q.; Bi, F. C.; Wang, Q. M. Bioorg. Med. Chem. 2007, 15, 3678.

[5] Ouyang, G. P.; Cai, X. J.; Chen, Z.; Song, B. A.; Bhadury, P. S.; Yang, S.; Jin, L. H.; Xue, W.; Hu, D. Y.; Zeng, S. J. Agric. Food Chem. 2008, 56, 10160.

[6] Hamaguchi, H.; Kajihara, O.; Katoh, M. J. Pestic. Sci. 1995, 20, 173.

[7] Ouyang, G. P.; Chen, Z.; Cai, X. J.; Song, B. A.; Bhadury, P. S.; Yang, S.; Jin, L. H.; Xue, W.; Hu, D. Y.; Zeng, S. Bioorg. Med. Chem. 2008, 16, 9699.

[8] Zhao, P. L.; Wang, L.; Zhu, X. L.; Huang, X. Q.; Zhan, C. G.; Wu, J. W.; Yang, G. F. J. Am. Chem. Soc. 2010, 132, 185.

[9] Song, H. J.; Liu, Y. X.; Xiong, L. X.; Li, Y. Q.; Yang, N.; Wang, Q. M. J. Agric. Food Chem. 2013, 61, 8730.

[10] Fu, C. R.; Peng, J.; Ning, Y.; Liu, M.; Shan, P. C.; Liu, J.; Li, Y. Q.; Hu, F. Z.; Zhu, Y. Q.; Yang, H. Z.; Zou, X. M. Pest. Manage. Sci. 2014, 70, 1207.

[11] Dai, H.; Liu, J. B.; Miao, W. K.; Wu, S. S.; Qin, X.; Zhang, X.; Wang, T. T.; Fang, J. X. Chin. J. Org. Chem. 2011, 31, 1662 (in Chinese).

(戴红, 刘建兵, 苗文科, 吴珊珊, 秦雪, 张欣, 王婷婷, 方建新, 有机化学, 2011, 31, 1662.)

[12] Dai, H.; Shi, L.; Zhang, H. J.; Li, Y. Q.; Fang, J. X.; Shi, Y. J. Chin. J. Org. Chem. 2012, 32, 1060 (in Chinese). (戴红, 施否, 张海军, 李永强, 方建新, 石玉军, 有机化学, 2012, 32, 1060.)

[13] Dai, H.; Yu, H. B.; Liu, J. B.; Qin, X.; Wang, T. T. Zhang, X.; Qin, Z. F.; Fang, J. X. Chin. J. Org. Chem. 2013, 33, 1104 (in Chinese). (戴红, 于海波, 刘建兵, 秦雪, 王婷婷, 张欣, 秦振芳, 方建新, 有机化学, 2013, 33, 1104.)

[14] Dai, H.; Xiao, Y. S.; Li, Z.; Xu, X. Y.; Qian, X. H. Chin. Chem.
Lett. 2014, 25, 1014

[15] Zhang, X.; Qin, Z. L. Chin. J. Org. Chem. 2006, 26, 870 (in Chinese).

(张欣，覃章兰，有机化学, 2006, 26, 870.)

[16] Li, Y. D.; Mao, W. T.; Fan, Z. J.; Li, J. J.; Fang, Z.; Ji, X. T.; Hua, X. W.; Zong, G. N.; Li, F. Y.; Liu, C. J.; Yu, J. H. Chin. Chem. Lett. 2013, 24, 1134.

[17] Wang, T. T.; Bing, G. F.; Zhang, X.; Qin, Z. F.; Yu, H. B.; Qin, X.; Dai, H.; Fang, J. X. Chin. J. Org. Chem. 2009, 29, 1287 (in Chinese).

(王婷婷，邴贵方，张欣，秦振芳，于海波，秦雪，戴红，方建新， 有机化学, 2009, 29, 1287.)

[18] Sun, Z. H.; Huang, W.; Gong, Y. Y.; Lan, J.; Liu, X. H.; Weng, J. Q.; Li, Y. S.; Tan, C. X. Chin. J. Org. Chem. 2013, 33, 2612 (in Chinese).

(孙召慧，黄伟，贡云芸，蓝健，刘幸海，翁建全，李永曙，谭成 侠, 有机化学, 2013, 33, 2612.)

[19] Wang, S. X.; Fang, Z.; Fan, Z. J.; Wang. D.; Li, Y. D.; Ji, X. T.; Hua, X. W.; Huang, Y.; Kalinina, T. A.; Bakulev, V. A.; Morzherin, Y. Y. Chin. Chem. Lett. 2013, 24, 889.

[20] Wang, Y. G.; Wang, Z. Y.; Zhao, X. Y.; Song, X. J. Chin. J. Org. Chem. 2004, 24, 1606 (in Chinese).

(汪炎钢, 王子云, 赵新等, 宋新建, 有机化学, 2004, 24, 1606.)

[21] Ling, Y.; Xiao, Y. A.; Chen, G. T.; Wang, D. G.; Li, Y. Q.; Wang, X. Y.; Zheng, H. Chin. Chem. Lett. 2012, 23, 1141.

[22] Cao, L.; Wang, Y. G.; Song, X. J.; Liu, G. H. Chin. J. Org. Chem. 2005, 25, 1007 (in Chinese).

(曹蕾, 汪炎钢, 宋新建, 刘国华, 有机化学, 2005, 25, 1007.)

[23] Yang, C.; Yang, S.; Song, B. A.; Hu, D. Y.; Chen, H. J.; Xue, W.; Jin, L. H.; Wu, J.; Xu, W. M.; Bai, S. Chin. J. Org. Chem. 2010, 30, 1327 (in Chinese).

(杨超, 杨松, 宋宝安, 胡德禹, 陈红军, 薛伟, 金林红, 吴剑, 徐维明, 柏松, 有机化学, 2010, 30, 1327.)

[24] Li, Y. J.; Liu, L. J.; Jin, K.; Sun, S. Q.; Xu, Y. T. Acta Chim. Sinica 2010, 68, 1577 (in Chinese).

(李英俊, 刘丽军, 靳焜, 孙淑琴, 许永廷, 化学学报, 2010, 68 , 1577.)

[25] Wang, T. T.; Bing, G. F.; Zhang, X.; Qin, Z. F.; Yu, H. B.; Qin, X.; Dai, H.; Miao, W. K.; Wu, S. S.; Fang, J. X. Bioorg. Med. Chem. Lett. 2010, 20, 3348 .

[26] Wu, S. S.; Miao, W. K.; Wang, T. T.; Fang, J. X. Chin. J. Org. Chem. 2015, 35, 1484 (in Chinese).

(吴珊珊, 苗文科, 王婷婷, 方建新, 有机化学, 2015, 35, 1484.)

[27] Wang, T. T.; Bing, G. F.; Zhang, X.; Qin, Z. F.; Yu, H. B.; Qin, X.; Dai, H.; Fang, J. X. Chem. J. Chin. Univ. 2010, 31, 708 (in Chinese).

(王婷婷，邴贵芳，张欣，秦振芳，于海波，秦雪，戴红，方建新， 高等学校化学学报, 2010, 31, 708.)

[28] Wang, S. L.; Shi, Y. J.; He, H. B.; Li, Y.; Li, Y.; Dai, H. Chin. Chem. Lett. 2015, 26, 672. 